\title{
Radiation from a Transmitting Aerial
}

$\mathrm{T}^{\mathrm{n}}$ HE transmitting aerial of a broadcasting station designed for local or national service should confine as much as possible of its radiated energy to the horizontal direction, emitting as little as possible in directions making an angle of less than $60^{\circ}$ with the vertical. The station will then have a large service area in which little fading is experienced. The demand for information on the design of such an aerial has given rise in recent years to some theoretical and experimental investigations on the general properties of antennæ used in radio transmitting stations.

A new and useful contribution to the state of knowledge on this subject has recently been given by Prof. P. O. Pedersen in a booklet published (in English) in Copenhagen ("Radiation from a Vertical Antenna over Flat Perfectly Conducting Earth." Pp. $49+35$ figures. G. E. C. Gad : Vimmelskaftet 32, Copenhagen. Kr. 6.00). This work comprises a theoretical investigation of the radiation characteristics of a vertical antenna over flat perfectly conducting earth, having for its objective a determination of the most suitable dimensions of the antenna at the new Copenhagen broadcasting station. The theoretical results have been checked by measurements of the distribution of the current in the antenna and of the field intensity at various distances from the station. To facilitate the theoretical analysis, the current distribution in the transmitting antenna is in the first case assumed to be sinusoidal, although it was known that this is not valid in the practical case. The complete general formulæ for the radiated field for an antenna are obtained, and a most useful series of tables and graphs are provided, from which the radiation resistance and the distribution of the radiated field in the vertical plane may be calculated for antennæ of various dimensions.

The author then applies the results to the practical case of the vertical antenna at the Copenhagen station. Measurements of the current and voltage distributions in this antenna have been made to ascertain to what extent they differ from the sinusoidal case. The chief point of difference is that the node of current at the base of the antenna, which is just over half a wavelength high, is replaced by a current minimum only. The formulæ are therefore recalculated to take account of this new distribution, and it is shown that the alteration produced in the radiation distribution diagram for the Copenhagen antenna is almost negligible.

The practical and very satisfactory result of the work described in this paper is, therefore, that for the type of half wave transmitting aerial which is now employed at many broadcasting stations, the radiation characteristics may be calculated with good approximation by the comparatively simple analysis based upon a sinusoidal distribution of current in the antenna.

\section{Work of the Bureau of Standards, Washington}

\begin{abstract}
LTHOUGH owing to the necessity for economy A the work of the U.S. Bureau of Standards had to be appreciably curtailed last year, yet the record of the work done as given in the annual report of the Secretary of Commerce (pp. 51-76) is impressive and interesting.
\end{abstract}

Valuable results were obtained in an experimental research on the transmission utility of the various frequencies used in broadcasting. The effects of ground waves and sky waves were determined. The sky waves were found to play a far more important role in distant daytime reception than was formerly supposed. The results obtained by measurements throughout the year on the height and ionisation of the conducting layers in the upper atmosphere which are responsible for long-distance radio transmission have greatly increased our knowledge of the subject. The relative effects produced by ultra-violet light, electrons and heavy ions have been determined.

Tests show that a new method of assembling watches having monometallic balance wheels and elinvar hairsprings has increased the uniformity of their time-keeping and has practically eliminated the deleterious effects that can be produced by magnetism. In association with the American Dental Association, the Bureau has developed and standardised methods for tarnish tests for dental alloys of low precious metal content. In connexion with criminal work, assistance was given in cases involving extortion, kidnapping, forgeries, threatening letters, etc.

The temperature of 'freezing' rhodium in vacuo has been found to be $1,966^{\circ} \mathrm{C}$. with a possible inaccuracy of $\pm 3^{\circ} \mathrm{C}$. The candle-power of tungsten filament lamp standards can now be found from the basic carbon filament standards. The vapour pressures of solid and liquid heavy hydrogen (deuterium) have been measured and from these data its freezing and boiling points and latent heats have been deduced. Interference measurements in the first spectra of several gases have been repeated and extended. Most of the spectral lines have been found reproducible to one part in fifty million and consequently are recommended as standards for spectroscopic and meteorological measurements.

Many useful hints are given amongst the results obtained. For example, tests made under carefully controlled conditions, with lighted cigarettes on grass and forest floor materials, show that the fire risk is greatly decreased when a cigarette paper tip $\frac{3}{4}-1$ in. long is applied in the course of manufacture over the end that is discarded. The possibility of utilising this result in practice is worth considering. The carpet wear-testing machine developed at the Bureau has been put to good use during the year. It has been found that the durability of a carpet is materially increased by increasing the density or the height of the pile and by the use of underlays. 